\title{
Disorder of the mevalonate pathway inhibits calcium-induced differentiation of keratinocytes
}

\author{
RUI JIN ${ }^{1}$, XIN LUO $^{1}$, KANG LUAN $^{1}$, LI LIU ${ }^{1}$, LIANG-DAN SUN ${ }^{2}$, \\ SEN YANG ${ }^{2}$, SHENG-QUAN ZHANG ${ }^{1,2}$ and XUE-JUN ZHANG ${ }^{2}$ \\ ${ }^{1}$ Department of Biochemistry and Molecular Biology; ${ }^{2}$ Department of Dermatology, Institute of \\ Dermatology, No. 1 Hospital, Anhui Medical University, Hefei, Anhui 230022, P.R. China
}

Received January 22, 2016; Accepted March 3, 2017

DOI: $10.3892 / \mathrm{mmr} .2017 .7128$

\begin{abstract}
Mutation of genes encoding the enzymes of the mevalonate pathway cause a variety of diseases, including skin disorders. Mutation of four genes in this pathway, including mevalonate kinase, phosphomevalonate kinase, mevalonate diphosphate decarboxylase and farnesyl diphosphate synthase, have demonstrated to be responsible for porokeratosis (PK). However, the pathogenesis of PK remains unclear. In the present study, specific enzyme inhibitors of the mevalonate pathway, including pravastatin (PRA), alendronate (ALD), farnesyl transferase inhibitor (FTI-277) and geranylgeranyl transferase inhibitor (GGTI-298), were used to investigate the effect on differentiation of keratinocytes (KCs). Western blotting demonstrated that PRA, ALD, FTI-277 or GGTI-298 alone, or in combination, inhibited the expression level of calcium-induced differentiation maker involucrin (INV) in KCs. ALD and PRA induced greater inhibition of INV compared with FTI-277 and GGTI-298 treatment. These inhibitors additionally influenced the expression levels of keratin1. Mechanistic studies revealed that treatment of cells with inhibitors decreased the expression levels of p53 and Notch1, and regulated activation of the mitogen activated protein kinase and phosphoinositide-3-kinase/protein kinase B signaling pathways. The results of the present study suggested that regulation of the mevalonate pathway may be necessary for differentiation of $\mathrm{KCs}$, and the pathogenesis of disseminated superficial actinic PK.
\end{abstract}

Correspondence to: Professor Sheng-Quan Zhang, Department of Biochemistry and Molecular Biology, Anhui Medical University, 81 Meishan Road, Hefei, Anhui 230022, P.R. China

E-mail:sqz36@yahoo.com

Professor Xue-Jun Zhang, Department of Dermatology, Institute of Dermatology, No. 1 Hospital, Anhui Medical University, 81 Meishan Road, Hefei, Anhui 230022, P.R. China

E-mail: ayzxj@vip.sina.com

Key words: keratinocytes, porokeratosis, disseminated superficial actinic, mevalonate pathway, differentiation

\section{Introduction}

The mevalonate metabolic pathway serves a key role in cellular processes, including proliferation, differentiation and apoptosis (1-3). Dysregulation of the pathway may lead to the development of diseases including cancer and cardiovascular disease $(4,5)$. Components of this pathway include mevalonic acid (MVA), isopentenyl pyrophosphate (IPP), ubiquinone (CoQ), farnesyl pyrophosphate (FPP), geranylgeranyl pyrophosphate, cholesterol(CH) and heme A (Fig. 1A) (6,7). It has been reported that mutation of genes coding for the enzymes in this pathway may lead to monogenic inherited diseases, including hyperimmunoglobulinemia D syndrome (HIDS) and porokeratosis (PK). The majority of these diseases exhibit lesions of the skin $(8,9)$.

The outer layer of skin, namely the epidermis, consists of keratinocytes (KCs), which are cells that differentiate and form a barrier against environmental damage. The skin is divided into layers, including the basal, spinous, granular and keratinization layers. In addition, the stratum lucidum layer is present in certain areas of the body, including on the palms of the hand and the soles of the feet. Each layer exhibits a gene expression profile that represents the level of differentiation (10). KCs in the basal layer possess high activity levels of keratin (K) 5 and 14. In the stratum spinosum, the expression levels of K5 and K14 are downregulated, whereas K1 and 10 are expressed. Following this, granular layer cells cease to express K1 and K10 and begin to produce late-differentiation products, including involucrin(INV) and loricrin. Previous studies have demonstrated that epidermal activity is associated with the mevalonate pathway (11). Pitavastatin, a HMG-CoA reductase inhibitor, has been demonstrated to induce phototoxicity in human keratinocytes, and fluvastatin may enhance the release of calcium ions $\left(\mathrm{Ca}^{2+}\right)$ to regulate keratinocyte function $(12,13)$. Pamidronate, an inhibitor of farnesyl diphosphate synthase (FDPS), induces senescence of human oral keratinocytes and expression of p63, and inhibits KC proliferation $(14,15)$.

Previous studies have reported that mutation of the mevalonate kinase $(M V K)$ gene may contribute to the phenotypes recognized in rare autosomal recessive diseases, including mevalonic aciduria and HIDS $(16,17)$. Our previous study identified that a heterozygous mutation of $M V K$ was 
responsible for cases of familial and sporadic disseminated superficial actinic porokeratosis (DSAP) (18). In addition, Zhang et al (19) identified mutations in three other genes of the mevalonate pathway ( $P M V K, M V D$ and $F D P S)$ in $98 \%$ familial and $73 \%$ sporadic PK patients. However, the pathological mechanism of PK and how the mevalonate pathway regulates this remains unclear. The present study used specific inhibitors of the mevalonate pathway and subsequently investigated the effect on $\mathrm{KC}$ differentiation.

\section{Materials and methods}

Chemicals and antibodies. Pravastatin(PRA), Alendonate (ALD), farnesyl transferase inhibitor (FTI-277) and geranylgeranyl transferase inhibitor (GGTI-298) were obtained from Sigma-Aldrich (Merck KGaA, Darmstadt, Germany). KC growth medium 154CF (M154) and coating matrix were purchased from Thermo Fisher Scientific, Inc. (Waltham, MA, USA). Anti-K1(mouse monoclonal $\mathrm{IgG}_{2 \mathrm{a}}$; sc-376224), involucrin (INV; rabbit polyclonal IgG; sc-28557), Notch1 (goat polyclonal IgG; sc-6014), p53 (rabbit polyclonal IgG; sc-6243), phosphorylated (p)-extracellular signal-regulated kinase (ERK; rabbit polyclonal IgG; sc-101761), p-protein kinase B (AKT; rabbit polyclonal IgG; sc-33437) and $\beta$-actin (mouse monoclonal IgG1; sc-376421) primary antibodies were purchased from Santa Cruz Biotechnology, Inc. (Dallas, TX, USA). The horseradish peroxidase conjugated secondary antibody (Rabbit anti-goat IgG, ZB-2306; goat anti-mouse $\mathrm{IgG}, \mathrm{ZB}-2305$; goat anti-rabbit IgG, ZB-2301) was provided by ZsBIO (OriGene Technologies, Beijing, China).

Cell culture. The present study was approved by Ethical Committee of Anhui Medical University (Anhui, China). Following the procurement of the informed consent from the participants, the surgery foreskin was collected from four volunteers at the Anhui Medical University and used to made the keratinocytes. Briefly, surgery foreskin was digested with $0.4 \%$ neutral protease at $4^{\circ} \mathrm{C}$ overnight. Subsequently, $0.025 \%$ trypsin (Sigma-Aldrich; Merck KGaA) was used to digest the epidermis, which was separated from the skin at $37^{\circ} \mathrm{C}$ for $\sim 5$ min to generate cell suspensions. Cells were cultured in $\mathrm{KC}$ growth medium $154 \mathrm{CF}$.

Western blot analysis. The KCs were cultured in 24-well dishes $\left(5 \times 10^{4} /\right.$ per well) at $37^{\circ} \mathrm{C}$ in $5 \% \mathrm{CO}_{2}$ atmosphere for $24 \mathrm{~h}$, following which the four inhibitors alone or in combination (PRA $1 \mu \mathrm{M}$, ALD $100 \mu \mathrm{M}$, FTI-277 $5 \mu \mathrm{M}$ and GGTI-298 $5 \mu \mathrm{M}$ ) were added into the medium. After $48 \mathrm{~h}$, the cells were treated with $1.2 \mathrm{mM} \mathrm{Ca}^{2+}$ for $24 \mathrm{~h}$, and then the total protein was extracted and used to analyze the differentiation markers expression. Briefly, cells were lysed using radioimmunoprecipitation assay buffer. Cellular lysate was centrifuged for $10 \mathrm{~min}$ at $12,000 \mathrm{x} \mathrm{g}$ at $4^{\circ} \mathrm{C}$ and the total protein concentration in each sample was analyzed using a Bicinchoninic Acid assay (Sigma-Aldrich; Merck KGaA). A total of $10 \mu \mathrm{g}$ proteins were loaded onto $12 \%$ SDS-polyacrylamide gels and subjected to electrophoresis. Proteins were subsequently transferred to polyvinylidene difluoride membranes (EMD Millipore, Billerica, MA, USA). Membranes were blocked in $5 \%$ non-fat milk for $2 \mathrm{~h}$ at room temperature and probed with primary antibodies against KI (1:500), INV $(1: 1,000)$, Notch1 (1:500), p53 (1:500), p-ERK (1:1,000), p-AKT (1:1,000) and $6 \beta$-actin $(1: 500)$ overnight at $4{ }^{\circ} \mathrm{C}$, followed by secondary antibodies(Rabbit anti-goat IgG 1:10,000; goat anti-mouse $\operatorname{IgG~1:5,000;~goat~anti-rabbit~IgG~1:5,000)~for~} 2 \mathrm{~h}$ at room temperature. Proteins were detected using chemiluminescence substrate (Pierce Biotechnology; Thermo Fisher Scientific, Inc.) and images were taken by the ChemiScope Imaging system (ClinxScience Instruments Co. Ltd., Shanghai, China) and densitometric analysis was performed using Quantity One software version 4.62 (Bio-Rad Laboratories, Inc., Hercules, CA, USA).

Statistical analysis. Data are expressed as the mean \pm standard error, and analyzed by one-way analysis of variance; multiple comparisons between the groups was performed using the S-N-K method. Experiments were repeated at least three times. Results were analyzed using SPSS software version 17.0 (SPSS, Inc., Chicago, IL, USA) and $\mathrm{P}<0.05$ was considered to indicate a statistically significant difference.

\section{Results}

Disruption of the mevalonate pathway reduces the expression levels of differentiation markers in KCsin the presence of $\mathrm{Ca}^{2+}$. Studies have demonstrated that the mevalonate pathway (Fig. 1A) may be associated with the expression of differentiation markers in KCs $(20,21)$. To investigate the role of the mevalonate pathway in $\mathrm{KC}$ differentiation, In the presence of $\mathrm{Ca}^{2+}$, treatment of cells with PRA, ALD, FTI-277 or GGTI-298 alone led to a reduction in the expression levels of INV $(\mathrm{P}<0.01$ vs. untreated control group in the presence of $\mathrm{Ca}^{2+}$; Fig. 1B). However, the inhibitory effect of PRA or ALD on INV expression was greater than that of FTI-277and GGTI-298. Treatment with two or three inhibitors in combination also resulted in a inhibitory effect on INV expression $(\mathrm{P}<0.01$ vs. untreated control group; Fig. 1C). In Fig. 1D, four inhibitors combination had a similar effect on expression of INV with the PRA or ALD. None of the inhibitors, either alone or in combination, significantly influenced the expression levels of $\mathrm{K} 1$.

Disruption of the mevalonate pathway reduces the expression levels of p53 and Notchl in KCs in the presence of $\mathrm{Ca}^{2+}$. Differentiation of KCs is a complex process that is regulated by a number of molecules $(22,23)$. Previous studies have demonstrated that $\mathrm{Ca}^{2+}$ stimulates the differentiation of KCs via the promotion of p53 expression and activation of Notch1 $(24,25)$. In the present study, the expression levels of p53 and Notch1 were measured in KCs treated with inhibitors of the mevalonate pathway. Treatment with PRA, ALD, FTI-277 or GGTI-298 reduced the expression levels of p53 and NCID, which is an activation form of Notch1 $(\mathrm{P}<0.01$ vs. untreated control group in the presence of $\mathrm{Ca}^{2+}$; Fig. 2A). When Notch1 binds with its ligand, it is cleaved to produce Notch1 extracellular truncation (NEXT), which is consequently cleaved to release notch1 intracellular domain (NCID) (26). In addition, in the presence or absence of $\mathrm{Ca}^{2+}$, treatment with two inhibitors in combination resulted in reduced expression levels of p53 and Notch1 $(\mathrm{P}<0.01$ vs. untreated control; Fig. $2 \mathrm{~B})$, as did treatment with 
A

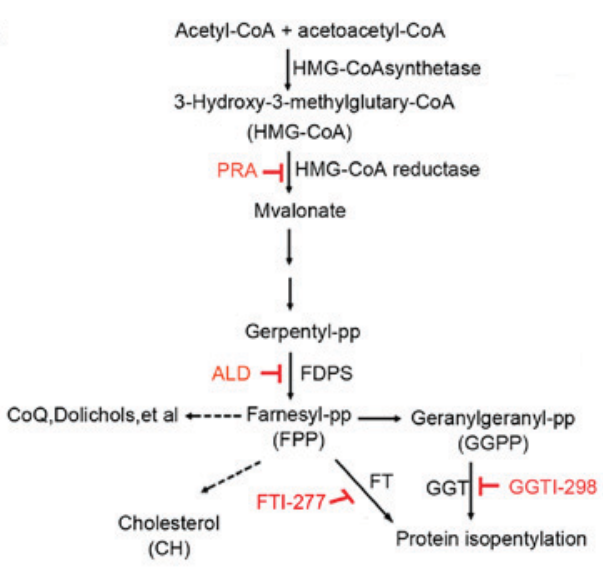

C

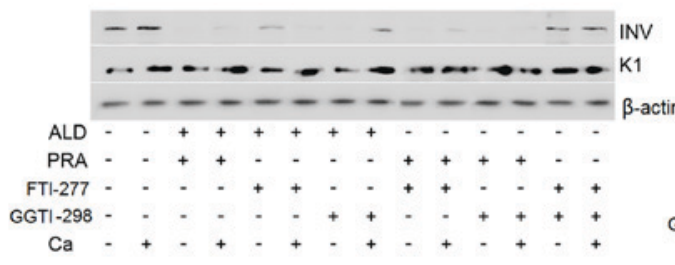

FTI-277

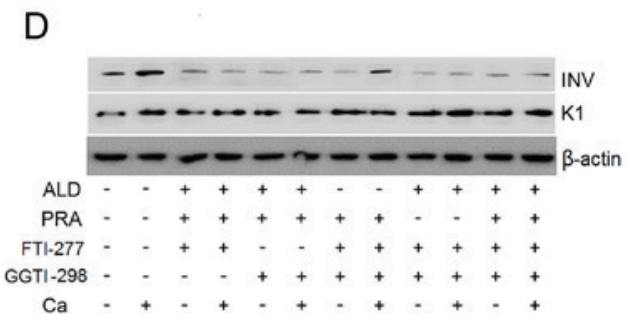

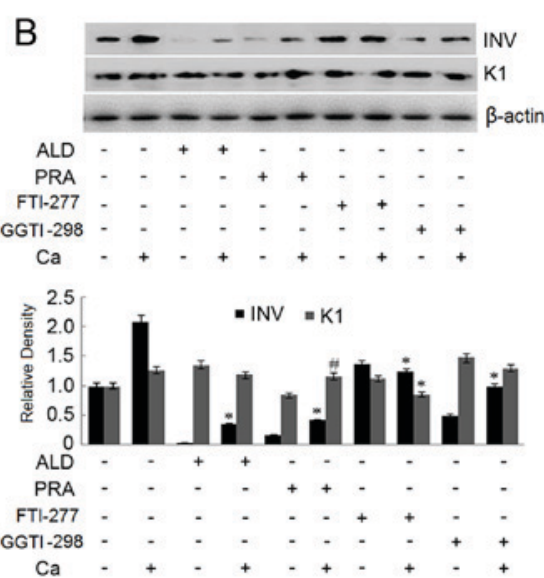

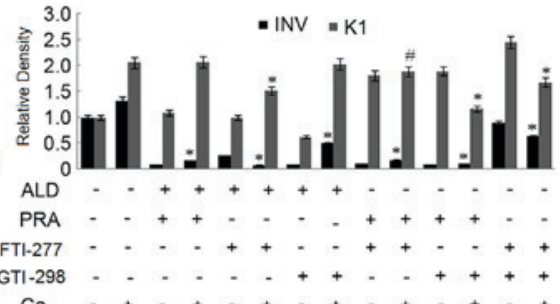

$\mathrm{Ca}-+\cdots+\cdots+\cdots+\cdots+\cdots+\cdots+$

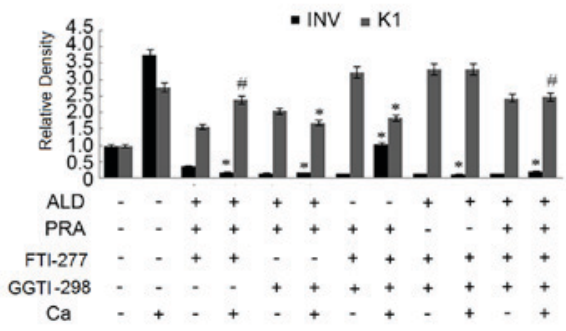

Figure 1. Treatment of KCs with inhibitors of the mevalonate pathway reduces the expression levels of differentiation markers in the presence of Ca ${ }^{2+}$ (A) Schematic of the mevalonate pathway. The sites of inhibition by PRA, ALD, FTI-277 and GGTI-298 are presented in red (B-D). Representative western blot images and quantification of protein expression levels of INV and K1 in KCs treated with PRA, ALD, FTI-277 and GGTI-298 as single agents or in combination for $48 \mathrm{~h}$, in the presence or absence of $\mathrm{Ca}^{2+}$. Data are expressed as mean \pm standard error of three independent experiments. ${ }^{*} \mathrm{P}<0.01$ and ${ }^{\#} \mathrm{P}<0.05 \mathrm{vs}$. untreated control in the presence of $\mathrm{Ca}^{2+}$.PRA, pravastatin; ALD, alendronate; FTI-277, farnesyl transferase inhibitor; GGTI-298, geranylgeranyl transferase inhibitor; INV, involucrin; K1, keratin1; KCs, keratinocytes.

three or four inhibitors in combination $(\mathrm{P}<0.01$ vs. untreated control; Fig. 2C).

Disruption of the mevalonate pathway affects the mitogen-activated protein kinase (MAPK) and phosphoinositide 3-kinase (PI3K)-AKT signaling pathways in the presence of $\mathrm{Ca}^{2+}$. Disruption of the mevalonate metabolic pathway affects the differentiation of KCs. In the present study, when the KCs was cultured without $\mathrm{Ca}^{2+}$, PLA or ALD increased the pAKT level. Single treatment reduced the level of pERK1/2 (Fig. 3A) and the combination treatment upregulated the level of $\mathrm{pAKT}$ compare with the control, but downregulated the level of pERK1/2 (Fig. 3B and C). When KCs were treated with $\mathrm{Ca}^{2+}$, only ALD enhanced the pAKT level, the others even demonstrating an inhibition effect on pAKT levels (Fig. 3A). Combination treatment of two inhibitors reduced the level of pAKT compared with the untreated control (Fig. 3B) but combinations of three or four inhibitors failed to influence the level of pAKT (Fig. 3C). On the contrary, the expression levels of pERK1/2 were not altered by single agent treatment (Fig. 3A) and were either unchanged or reduced by combination treatment (Fig. 3B and C).

\section{Discussion}

The mevalonate pathway serves a role in the physical processes of cells, including KCs. In the present study, specific inhibitors were used to investigate the effect of the mevalonate pathway on KCs. Four inhibitors inhibited the INV differentiation maker in KCs; however, PRA and ALD had a greater effect than FTI-277 and GGTI-298. Inhibitor treatment (singly or in combination) reduced the expression of p53 and Notch1 and regulated the activation of PI3K-AKT and MAPK pathways in KCs.

PRA may cause a shortage in downstream products of MVA, whereas ALD may cause an accumulation of MVA, IPP, dimethylallyl diphosphate and N6-isopentenyladenosine, in addition to a shortage of downstream products from FPP. Therefore, when KCs were treated with ALD in combination with PRA, PRA may have reduced the expression levels of 

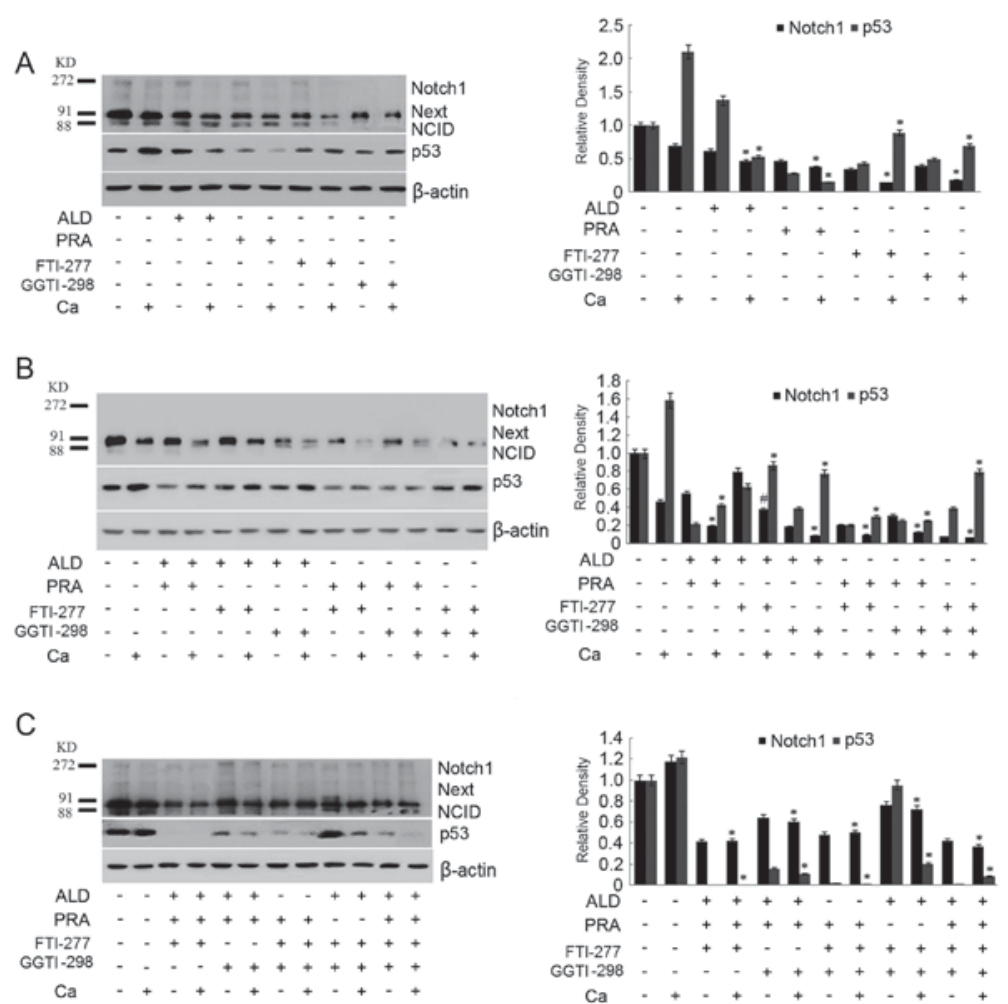

Figure 2. Treatment of KCs with inhibitors of the mevalonate pathway reduced the expression levels of Notch1 and p53. Representative western blot images and quantification of protein expression levels of Notch1 and p53 in KCs treated with PRA, ALD, FTI-277 and GGTI-298 inhibitors (A) alone, (B) in combination with two inhibitors or (C) in combination with three or four inhibitors for $48 \mathrm{~h}$, in the presence or absence of $\mathrm{Ca}^{2+}$. Data are expressed as mean \pm standard error of three independent experiments. ${ }^{*} \mathrm{P}<0.01$ and ${ }^{~} \mathrm{P}<0.05$ vs. untreated control in the presence of $\mathrm{Ca}^{2+}$. PRA, pravastatin; ALD, alendronate; FTI-277, farnesyl transferase inhibitor; GGTI-298, geranylgeranyl transferase inhibitor; KCs, keratinocytes; NEXT, Notch1 extracellular truncation; NCID, Notch1 intracellular domain.
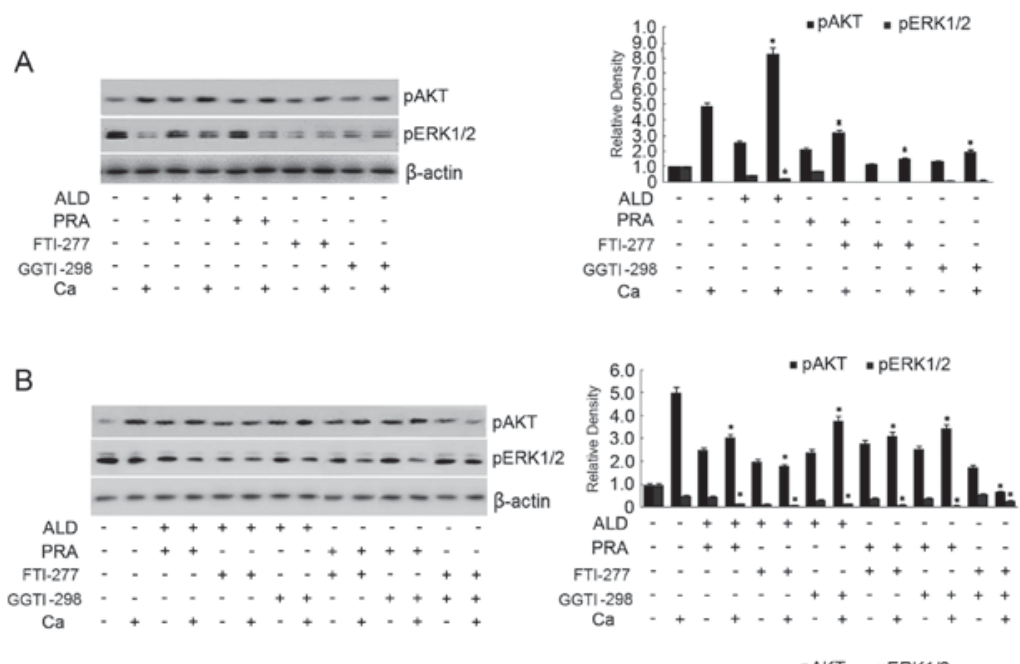

C

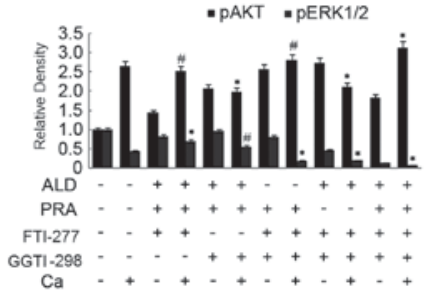

Figure 3. Treatment of KCs with inhibitors of the mevalonate pathway alters the expression levels of components of the mitogen activated protein kinase and phosphoinositide 3-kinase-AKT signaling pathways. Representative western blot images and quantification of p-AKT and p-ERK1/2 protein expression levels in KCs treated with PRA, ALD, FTI-277 and GGTI-298 inhibitors (A) alone, (B) in combination with two inhibitors or (C) in combination with three or four inhibitors for $48 \mathrm{~h}$, in the presence or absence of $\mathrm{Ca}^{2+}$. Data are expressed as mean \pm standard error of three independent experiments. ${ }^{*} \mathrm{P}<0.01$ and ${ }^{~} \mathrm{P}<0.05$ vs. untreated control in the presence of $\mathrm{Ca}^{2+}$. p, phosphorylated; AKT, protein kinase B; PRA, pravastatin; ALD, alendronate; FTI-277, farnesyl transferase inhibitor; GGTI-298, geranylgeranyl transferase inhibitor; ERK1/2, extracellular signal-regulated kinase; KCs, keratinocytes. 
MVA, and partially reduced the effect of ALD on KCs, which caused an accumulation of MVA. However, in the present study, this phenomenon was not observed. $\mathrm{CH}$ has been reported to promote the expression levels of differentiation markers in KCs. These results suggested that an active mevalonate pathway in $\mathrm{KCs}$ facilitates differentiation, in particular, $\mathrm{CH}$ synthesis $(8,20)$. In addition, the inhibitors reduced the expression levels of p53 and Notch1, which are involved in the differentiation of KCs (24). Notch1 functions as a receptor for membrane-bound ligands (jagged1, jagged2 and delta1) to regulate cell differentiation, proliferation and apoptotic processes. When Notch1 binds with its ligand, it is cleaved to produce NEXT, which is consequently cleaved to release NCID. Then it forms a transcriptional activator complex with recombination signal binding protein for immunoglobulin $\kappa \mathrm{J}$ region and activates genes to regulate the cell fate (26). However, differentiation of $\mathrm{KCs}$ is a complex process that is regulated by a number of molecules $(22,23)$. Therefore, the results of the present study partly explain the underlying mechanism of differentiation inhibition caused by dysfunction of the mevalonate pathway. None of the inhibitors exhibited an effect on the expression levels of $\mathrm{K} 1$ in the presence of $\mathrm{Ca}^{2+}$, as $\mathrm{K} 1$ is an early differentiation marker. Generally, when $\mathrm{KCs}$ were treated with $\mathrm{Ca}^{2+}$, changes in $\mathrm{K} 1$ expression occur in $12 \mathrm{~h}$. As the KCs in the present study were treated with $\mathrm{Ca}^{2+}$ for $24 \mathrm{~h}$, it may explain why changes in $\mathrm{K} 1$ expression were not observed. The change of late-differentiation marker INV was marked, suggesting that KCs differentiation was into late-stage, but further studies are required for confirmation.

In the present study, inhibitors of the mevalonate pathway regulated the activation of PI3K-AKT and MAPK pathways in $\mathrm{KCs}$ in different ways. Previous studies have demonstrated that $\mathrm{Ca}^{2+}$ promoted the KCs differentiation through increasing the level of pAKT and reducing the level of pERK1/2 (24). Thus, although the inhibitors upregulated the level of pAKT compared with the control (without $\mathrm{Ca}^{2+}$ ), no significant effect on level of pAKT was observed, except in the case of ALD; this may be due to the stronger effect of $\mathrm{Ca}^{2+}$ on pAKT than the inhibitors. For the same reason, the inhibitors did not exhibit an influence on the level of pERK1/2. It is notable that the different inhibitors (singly or in combination) exerted different effects on the level of pAKT and pERK1/2; this may be attributed to the target steps of the inhibitors, which cause different accumulations of the intermediates and a shortage of the downstream products. These results suggested that the underlying molecular mechanisms of differentiation are complex.

In conclusion, dysfunction of mevalonate pathway may inhibit the differentiation of KCs to different degrees. Therefore, efficient regulation of the mevalonate pathway is essential for $\mathrm{KC}$ differentiation. These results suggested that the underlying pathological mechanism of DSAP requires further investigation.

\section{Acknowledgements}

The present study was supported by the General Program of National Natural Science Foundation of China (grant no. 81271748) and the University Science Research Project of Anhui Province (grant no. KJ2017A195).

\section{References}

1. Zanette DL, Lorenzi JC, Panepucci RA, Palma PV, Dos Santos DF, Prata KL and Silva WA Jr: Simvastatin modulates mesenchymal stromal cell proliferation and gene expression. PLoS One 10: e0120137, 2015.

2. Olivera-Martinez I, Schurch N, Li RA, Song J, Halley PA, Das RM, Burt DW, Barton GJ and Storey KG: Major transcriptome re-organisation and abrupt changes in signalling, cell cycle and chromatin regulation at neural differentiation in vivo. Development 141: 3266-3276, 2014.

3. Ghavami S, Sharma P, Yeganeh B, Ojo OO, Jha A, Mutawe MM, Kashani HH, Los MJ, Klonisch T, Unruh $\mathrm{H}$ and Halayko AJ: Airway mesenchymal cell death by mevalonate cascade inhibition: Integration of autophagy, unfolded protein response and apoptosis focusing on Bcl 2 family proteins. Biochim Biophys Acta 1843: 1259-1271, 2014.

4. Yeganeh B, Wiechec E, Ande SR, Sharma P, Moghadam AR, Post M, Freed DH, Hashemi M, Shojaei S, Zeki AA and Ghavami S: Targeting the mevalonate cascade as a new therapeutic approach in heart disease, cancer and pulmonary disease. Pharmacol Ther 143: 87-110, 2014.

5. Fearon KC, Glass DJ and Guttridge DC: Cancer cachexia: Mediators, signaling, and metabolic pathways. Cell Metab 16: 153-166, 2012 .

6. Miziorko HM: Enzymes of the mevalonate pathway of isoprenoid biosynthesis. Arch Biochem Biophys 505: 131-143, 2011.

7. Buhaescu I and Izzedine H: Mevalonate pathway: A review of clinical and therapeutical implications. Clin Biochem 40: 575-584, 2007.

8. Horvat S, McWhir J and Rozman D: Defects in cholesterol synthesis genes in mouse and in humans: Lessons for drug development and safer treatments. Drug Metab Rev 43: 69-90, 2011.

9. Haas D and Hoffmann GF: Mevalonate kinase deficiencies: From mevalonic aciduria to hyperimmunoglobulinemia D syndrome. Orphanet J Rare Dis 1: 13, 2006.

10. Fuchs E: Epidermal differentiation: The bare essentials. J Cell Biol 111: 2807-2814, 1990.

11. Feingold KR: The regulation and role of epidermal lipid synthesis. Adv Lipid Res 24: 57-82, 1991.

12. Viola G, Grobelny P, Linardi MA, Salvador A, Dall'Acqua S, Sobotta $Ł$, Mielcarek J, Dall'Acqua F, Vedaldi D and Basso G: Pitavastatin, a new HMG-CoA reductase inhibitor, inducesphototoxicity in human keratinocytes NCTC-2544 through the formation of benzophenanthridine-like photoproducts. Arch Toxicol 86: 483-496, 2012.

13. Coutant KD, Wolff-Winiski B and Ryder NS: Fluvastatin enhances receptor-stimulated intracellular $\mathrm{Ca} 2+$ release in human keratinocytes. Biochem Biophys Res Commun 245: 307-312, 1998.

14. Kim RH, Lee RS, Williams D, Bae S, Woo J, Lieberman M, Oh JE, Dong Q, Shin KH, Kang MK and Park NH: Bisphosphonates induce senescence in normal human oral keratinocytes. J Dent Res 90: 810-816, 2011.

15. Scheller EL, Baldwin CM, Kuo S, D'Silva NJ, Feinberg SE, Krebsbach PH and Edwards PC: Bisphosphonates inhibit expression of p63 by oral keratinocytes. J Dent Res 90: 894-899, 2011.

16. Lainka E, Neudorf U, Lohse P, Timmann C, Bielak M, Stojanov S, Huss $\mathrm{K}$, von Kries R and Niehues T: Incidence and clinical features of hyperimmunoglobulinemia $D$ and periodic fever syndrome (HIDS) and spectrum of mevalonate kinase (MVK) mutations in German children. Rheumatol Int 32: 3253-3260, 2012.

17. Stoffels M and Simon A: Hyper-IgD syndrome or mevalonate kinase deficience. Curr Opin Rhumatol 23: 419-423, 2011

18. Zhang SQ, Jiang T, Li M, Zhang X, Ren YQ, Wei SC, Sun LD, Cheng H, Li Y, Yin XY, et al: Exome sequencing identifies MVK mutations in disseminated superficial actinic porokeratosis. Nat Genet 44: 1156-1160, 2012.

19. Zhang Z, Li C, Wu F, Ma R, Luan J, Yang F, Liu W, Wang L, Zhang S, Liu Y, et al: Genomic variations of the mevalonate pathway in porokeratosis. Elife 4: e06322, 2015.

20. Hanyu O, Nakae H, Miida T, Higashi Y, Fuda H, Endo M, Kohjitani A, Sone $\mathrm{H}$ and Strott CA: Cholesterol sulfate induces expression of the skin barrier protein filaggrin in normal human epidermal keratinocytes through induction of ROR $\alpha$. Biochem Biophys Res Commun 428: 99-104, 2012. 
21. Vukelic S,Stojadinovic O,PastarI,Vouthounis C,Krzyzanowska A, Das S, Samuels HH and Tomic-Canic M: Farnesyl pyrophosphate inhibits epithelialization and wound healing through the glucocorticoid receptor. J BiolChem 285: 1980-1988, 2010.

22. Botchkarev VA, Gdula MR, Mardaryev AN, Sharov AA and Fessing MY: Epigenetic regulation of gene expression in keratinocytes. J Invest Dermatol 132: 2505-2521, 2012.

23. Popp T, Steinritz D, Breit A, Deppe J, Egea V, Schmidt A, Gudermann T, Weber C and Ries C: Wnt5a/ $\beta$-catenin signaling drives calcium-induced differentiation of human primary keratinocytes. J Invest Dermatol 134: 2183-2191, 2014.

24. Fisher B, Metzger M, Richardson R, Knyphausen P, Ramezani T, Franzen R, Schmelzer E, Bloch W, Carney TJ and Hammerschmidt M: p53 and TAp63 promote keratinocyte proliferation and differentiation in breeding tubercles of the zebrafish. PLoS Genet 10: e1004048, 2014.
25. Eckert RL, Crish JF, Efimova T, Dashti SR, Deucher A, Bone F, Adhikary G, Huang G, Gopalakrishnan R and Balasubramanian S: Regulation of involucrin gene expression. J Invest Dermatol 123: 13-22, 2001.

26. Brutsh R, Liebler SS, Wüstehube J, Bartol A, Herberich SE, Adam MG, Telzerow A, Augustin HG and Fischer A: Integrin cytoplasmic domain-asssociatied protein-1 attenuates sprouting angiogenesis. Circ Res 107: 592-601, 2010. 